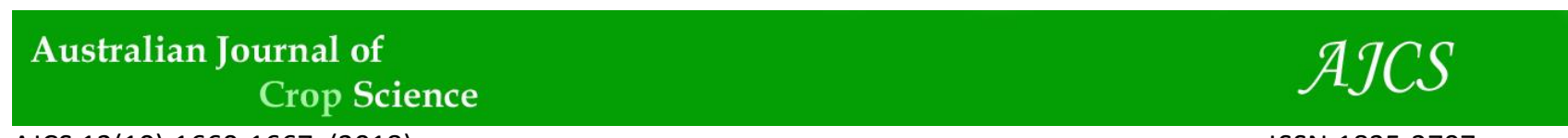

AJCS 12(10):1660-1667 (2018)

ISSN:1835-2707

doi: 10.21475/ajcs.18.12.10.pne1377

\title{
Comparative analysis of the Agrobacterium mediated transformation using primary and secondary callus of indica rice (Oryza sativa L.) using phosphinothricin as selecting medium
}

\author{
Pravin Kumar, Ishwar Chand, Ram Singh Purty
}

Pravin Kumar, University School of Biotechnology, Guru Gobind Singh Indraprastha University, Sector-16C, Dwarka, New Delhi-110078, India

Ishwar Chand, Department of Plant Molecular Biology, University of Delhi South Campus, New Delhi-110021, India

*Corresponding author: rspurty@ipu.ac.in

\begin{abstract}
Inefficiency of the Agrobacterium-mediated transformation in indica rice cultivar has been widely reported in the literature. Till date, mature seeds, primary and secondary calli have been the mostly used explants for transformation in indica rice. Therefore, the present investigation was undertaken to compare the Agrobacterium mediated transformation using primary and secondary callus as explants in the indica rice and testing a herbicide phosphinothricin (PPT) based bar gene selection system. Four different constructs containing the bar gene expression cassette were made. The constructs I and II were transformed with the secondary calli based protocol by three independent experiments. Similarly, constructs III and IV were transformed with the primary calli based protocol by 9 and 20 independent experiments, respectively. The transformation efficiency for the primary and secondary calli based transformation under PPT selection were calculated in the range of $0.13-0.26 \%$ and $2.0-2.66 \%$, respectively. The transgenic escapes were detected by genomic DNA PCR amplification, Southern blot analysis and basta leaf painting assay. It was found that the primary calli based transformation has 46-50\% transgenic escapes, whereas secondary calli based transformation have only $20-29 \%$ transgenic escapes. The present study reports that secondary calli as explants perform better than primary calli under PPT selection in the Agrobacterium-mediated indica rice transformation.
\end{abstract}

Keywords Agrobacterium tumefaciens; Phosphinothricin; bar gene; Secondary calli; Primary calli; Transgenic efficiency; Transgenic escape.

Abbreviations: PPT_Phosphinothricin.

Introduction

Rice is an excellent crop model plant for the molecular biology and genomics studies. The traditional plant breeding practice alone cannot fulfill the huge food demand for the ever-increasing world population. So, there is an urgent need for newer and better ways to achieve enhanced crop productivity. One excellent opportunity is being provided to the breeder through breakthrough techniques of genome editing. But these techniques very much rely on genetic transformation and regeneration protocols. Thus, genetic transformation and regeneration could be an integral tool for modern breeding strategies.

Plant transformation and regeneration has been a backbone for the fundamental insights into plant biology and in revolutionizing commercial agriculture, but for most of the crops, it remains a difficult task even after more than 30 years of technological advances (Altpeter et al., 2016). The Agrobacterium-mediated transformation, which accounts for more than $80 \%$ of plant genetic transformation, has many benefits over the other methods of transformations e.g. the ease and low cost, high efficiency of transformation, easy transfer of large segments of DNA with defined ends, the low copy number of transgenes inserted into the host genome etc. (Hiei et al., 1994, 2014; Hiei and Komari, 2008).
The genetic transformation protocols using the Agrobacterium were widely established for several different genotypes with selection marker either with hygromycin (Hiei et al., 1994) or phosphinothricin (PPT, commercial formulation 'Basta') (Toki et al., 1992) and still continue to represent two-dominant selection medium (Chen et al., 2010). The bar gene marker system based on PPT selection are supposed to be fast, cheap and easy utilization for screening of transformants in both in-vitro and field conditions (Khuong et al., 2013).

The Agrobacterium-mediated rice genetic transformation efficiency is determined by many factors but types and stages of explants are most important. The duration of mature seed-derived callus, which is being taken as explant, is most critical parameter for optimizing the transformation efficiency. There are two types of mature seed-derived callus: primary callus, i.e., less than 10 -days old callus which represents proliferation of scutellum and secondary callus, i.e., more than 2-week-old callus proliferating from primary callus (Saika and Toki 2010).

Hiei et al., (1994) established the highly efficient Agrobacterium-mediated transformation system using the mature seed-derived secondary calli as the explant with 
around $30 \%$ transformation efficiency in japonica rice, which generally takes 3-4 months to transfer plantlets into the soil. This protocol with some modifications is still being used for generation of japonica transgenic rice with transformation efficiency up to $70 \%$ with hygromycin selection (Saika and Toki 2010; Sun et al., 2017; Lin et al., 2018). This protocol became the most common rice transformation method for japonica rice; but not for indica rice, which accounts for approximately $80 \%$ of the cultivated rice (Mahesh et al., 2016). Despite lower transformation efficiency, this protocol with some modifications is still being used for generation of the indica transgenic rice vis-a-vis transformation efficiency is in the range of $0.8-46 \%$ with hygromycin (Lin and Zhang 2004; Sahoo et al., 2011; Mannimaran et al,. 2013; Reddy et al., 2016; Rao et al., 2017) and 0.56- $1.95 \%$ with PPT (Ramesh et al., 2004) selection depending upon different experimental conditions.

The transformation efficiency with PPT seems to be very poor than hygromycin selection. Ramesh et al., (2004) has reported very low transformation efficiency (between 0.56 $1.95 \%)$ with secondary calli based transformation under PPT selection in six indica rice cultivars with around 30\% transgenic escapes. Dedicova et al. (2014) has reported around $0.13-1.88 \%$ transgenic efficiency and $40-45 \%$ transgenic escapes after 10 different constructs transformation in Brazilian Curinga japonica rice by secondary calli based transformation under PPT selection. The bar gene-based selection system produced numerous herbicide-resistant biotech crops because its public acceptability [ $53 \%$ of biotech crop planted globally has herbicide tolerance (ISAAA GM Approval database, 2015)], but, if we analyze the published reports based on bar gene based rice genetic transformations for other regular molecular genomic functional studies, very few examples are available in literature. More than $90 \%$ published reports of rice transgenics are based on hygromycin selection (Sah et al., 2014; Singh and Prasad, 2016). This difference clearly indicates associated problems with PPT selection in terms of transformation efficiency and transgenic escapes during rice transformation and regeneration.

The continuous advancement in plant transformation techniques have been reported since its inception, but one major technological breakthrough was the use of primary callus instead of secondary callus as explant (Toki et al., 2006). The primary callus based transformation method has reported 95-98\% transformation efficiency with hygromycin selection in japonica rice, which takes only 45 days to transfer plantlets into soil (Toki et al., 2006). Since this primary callus based rice transformation with hygromycin selection has very high transformation efficiency with almost half transformation duration, most plant scientists now prefer genetic transformation based on primary over secondary callus to save precious time and resources. Now, many reports of indica rice transformation is also coming based on primary callus with hygromycin selection (Nguyen et al., 2017; Mehra et al., 2017; Burman et al., 2018). However, there is no report with PPT herbicide selection. Thus, there is need to compare the differences in the Agrobacterium transformation efficiency between secondary (Hiei et al., 1994) and primary callus (Toki et al., 2006) based transformation protocol with PPT (bar gene) selection in the indica rice. Here, we are reporting our interesting finding with PPT selection in the indica rice cultivar PB1.

\section{Results}

Development of transforming binary vectors

Four different constructs were developed for the present study in pPZP200 binary vector as described in the material and methods (Fig 1). All the constructs had the bar gene expression cassette for both in-vitro and in field selection.

\section{Transformation efficiency}

The constructs I and II were transformed with the secondary calli based protocol (Hiei et al. 1994) with some modifications as discussed earlier. The transformation efficiency was calculated as independent transgenic plants obtained / number of explants used for co-cultivation. The total 14 independent putative transgenic lines with construct I were obtained from 3 different experiments with transformation efficiency in the range of 2.0 - 2.5 (Table 1). Similarly, 23 independent putative transgenic lines with construct II were obtained from 3 different experiments with transformation efficiency in the range of $2.3-2.6$ (Table 1).

The constructs III and IV were transformed with the primary calli based on Toki et al., (2006) protocol with some modifications. Five independent putative transgenic lines with construct III were obtained from 9 different experiments with transformation efficiency in the range of $0.13-0.21$ (Table 1). Similarly, 15 independent putative transgenic lines with construct IV were obtained from 20 different experiments with transformation efficiency in the range of $0.19-0.26$ (Table 1 ).

\section{Transgenic escapes}

Molecular analysis was done to find the true transgenic lines growing in green house. PCR amplification of T-DNA genomic fragments towards the left border (LB) or the right border (RB) was done with respective forward and reverse primers as shown in Fig 1 . The T-DNA genomic fragment towards LB from all the four constructs was amplified with primer 1 and 2 (Supplementary Table 1). The T-DNA genomic fragment towards RB of construct I and IV was amplified with primer 3 and 4 whereas for construct II and III, primer 5 and 4 were used (Supplementary Table 1). Out of 5 (construct I), 17 (construct II), 2 (construct III) and 13 (construct IV) $\mathrm{T}_{0}$ surviving transgenic lines growing in green house $4,12,1$ and 7 lines have amplified both T-DNA genomic fragments, respectively (Fig 4). The PCR amplification result showed that putative transgenic lines obtained from construct I, II, III and IV have $20 \%, 29 \%, 50 \%$ and $46 \%$ transgenic escapes, respectively (Fig 4, Table 1 ).

The transgenic escapes were further checked by Southern blot analysis in some of the transgenic lines (Fig 5). The genomic DNA digested with Sbfl (construct I and II), Sacl (construct III) and $\mathrm{Ncol}$ (construct IV) enzymes were probed with bar gene specific probe to identify the T-DNA - genomic DNA junction in transgenic lines (Fig 1). The transgenic lines lacking PCR amplification specifically lacked any visualization of bands in Southern analysis, confirming transgenic escapes (Fig 5).

The transgenic escapes were finally confirmed by Basta leaf 
Table 1. The difference of transformation efficiency and escapes of different constructs in secondary and primary calli based Agrobacterium transformation.

\begin{tabular}{|c|c|c|c|c|c|c|c|}
\hline Construct Used & $\begin{array}{l}\text { Number of } \\
\text { Experiment } \\
\mathrm{s}\end{array}$ & $\begin{array}{l}\text { Number of } \\
\text { calli } \\
\text { transferred on } \\
\text { selection } \\
\text { medium }\end{array}$ & $\begin{array}{l}\text { Age of the } \\
\text { calli used for } \\
\text { transformati } \\
\text { on }\end{array}$ & $\begin{array}{l}\text { Number of } \\
\text { independent } \\
\text { transgenic } \\
\text { plants } \\
\text { obtained }\end{array}$ & $\begin{array}{l}\text { Transformatio } \\
\text { n efficiency } \\
\text { (\%) }\end{array}$ & $\begin{array}{l}\text { Percent } \\
\text { Transgenic } \\
\text { escapes (Plants } \\
\text { analyzed) }\end{array}$ & Transformation method \\
\hline Construct I & 3 & $\sim 600$ & $21 \mathrm{~d}$ & 14 & $2.0-2.5$ & $20(5)$ & \multirow{2}{*}{$\begin{array}{l}\text { Hiei et al., } 1994 \text { with few } \\
\text { modification }\end{array}$} \\
\hline Construct II & 3 & $\sim 900$ & $21 \mathrm{~d}$ & 23 & $2.33-2.66$ & $29(17)$ & \\
\hline Construct III & 9 & $\sim 2700$ & $05 \mathrm{~d}$ & 5 & $0.13-0.21$ & $50(2)$ & \multirow{2}{*}{$\begin{array}{l}\text { Toki et al., } 2006 \text { with few } \\
\text { modification }\end{array}$} \\
\hline Construct IV & 20 & $\sim 6000$ & $05 \mathrm{~d}$ & 15 & $0.19-0.26$ & $46(13)$ & \\
\hline
\end{tabular}

A

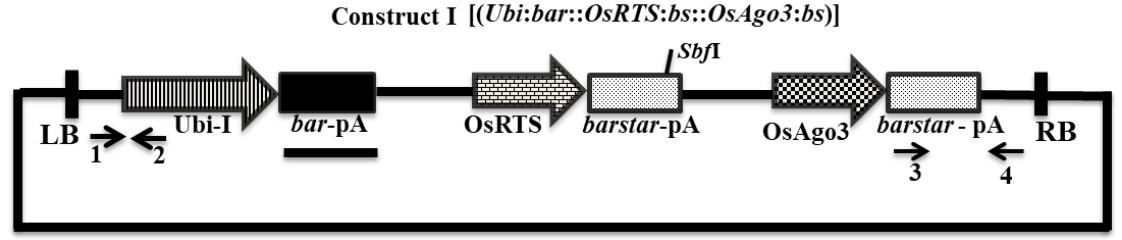

B

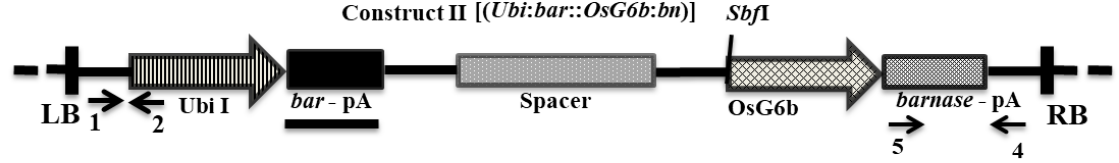

C

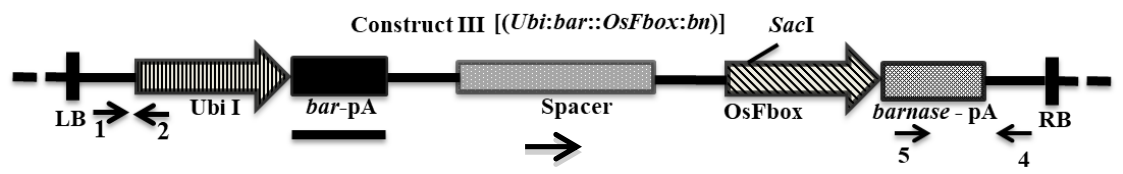

D

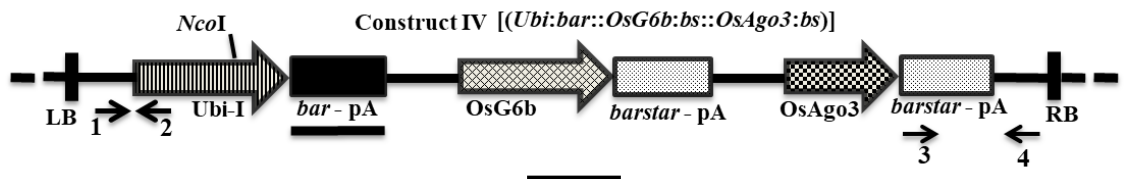

Fig 1. Schematic representation of the T-DNA regions of four different constructs used for the indica rice transformations study. (A) Construct I (Ubi:bar::OsRTS:bs::OsAgo3:bs). (B) Construct II (Ubi:bar::OsG6b:bn). (C) Construct III (Ubi:bar::OsFbox:bn). (D) Construct IV (Ubi:bar::OsG6b:bs::OsAgo3:bs). It is also showing the positions of various primers $(\rightarrow)$ that were used for PCR amplification to check the putative transgenic lines. The probe ( $)$ and enzyme used for digestion for southern blot analysis is also shown. The location of the enzymes used for genomic DNA digestion during Southern blot analysis is also indicated in T-DNA map. [LB - left border end of T-DNA, RB - right border end of T-DNA, Ubi-l- Ubiquitin promoter, pA- poly A signal sequence, $b s$ barstar gene, bn - barnase gene. The direction of Ubi-I, OsRTS, OsAgo3, OsG6b, and OsFbox promoters are indicated as arrow. No DNA sequence gap is present between two cassettes in any constructs and the bold lines between two cassettes are only for beautiful representation of cartoon].

painting assay, where the positive transgenic lines have shown no yellowing of leaves and transgenic escapes have turned yellow (Fig 6 showing result of cassette IV, results of other constructs are not shown here).

\section{Discussion}

Any advancement in the rice transformation and regeneration protocol has always been a welcome step among plant scientists. The secondary callus based Hiei et al. (1994) and primary callus based Toki et al., (2006) transformation methods are being used even in indica rice with hygromycin selection, but reports regarding PPT selection are limited. The present work was initiated to compare the differences in the Agrobacterium transformation efficiency between secondary and primary calli based transformation methods with PPT (bar gene) selection in the indica rice.
Four different constructs were made and transformations were done in the PB1 indica rice cultivar. Constructs I and II were transformed into secondary and constructs III and IV into primary calli by Hiei et al. (1994) and Toki et al. (2006) method, respectively. Secondary calli based transformation have $2.0-2.66 \%$ transformation efficiency with $20-29 \%$ transgenic escapes whereas primary calli based transformations have $0.13-0.26 \%$ transformation efficiency with $46-50 \%$ transgenic escapes in PPT selection (Table 1). The transformation efficiency is almost 10 times more and transgenic escapes are almost half in case of secondary calli than primary calli based transformation. We had tried to increase the transformation efficiency by increasing PPT concentration in selection and regeneration medium from $2.0 \mathrm{mgl}^{-1}$ to $8.0 \mathrm{mgl}^{-1}$ in primary calli based transformation, but there is no enhancement in transformation efficiency. The problem either in $2.0 \mathrm{mgl}^{-1}$ or $8.0 \mathrm{mgl}^{-1}$ PPT was similar i.e. the calli were not able to regenerate due to necrosis and ultimately died during second or third regeneration medium. 

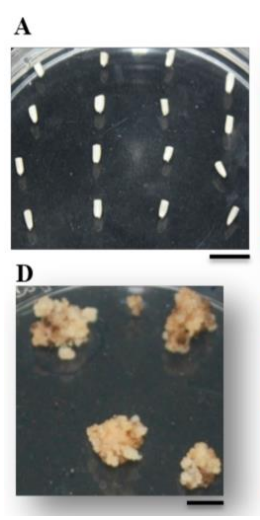

B
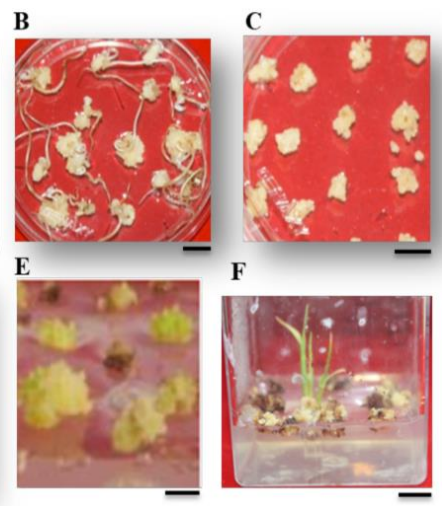

Fig 2. Representative images of secondary calli based rice transformation with bar gene selection. (A) Inoculation of dehusked and sterilized seed on CIM medium. (B) 21 days old secondary calli on CIM medium after incubation in dark at $26^{\circ} \mathrm{C}$. (C) Subcultured calli pre-incubated on callus maintenance medium (CMM) before Agrobacterium transformation. (D) Transformed calli on Selection medium after 45 days of incubation. (E) Regeneration of calli after 30 days of incubation on regeneration medium. (F) Plantlet coming out from the green regenerating calli after 45 days of incubation on regeneration medium (Scale bar $\sim 1.0 \mathrm{~cm}$ ).

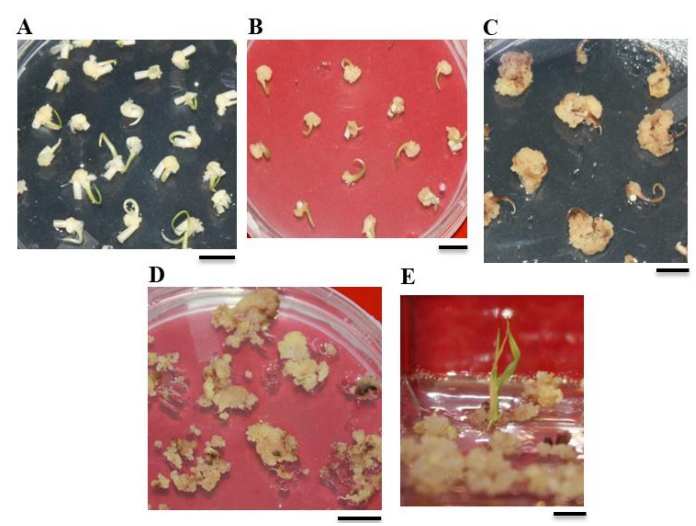

Fig 3. Representative images of primary calli based rice transformation with bar gene selection. (A) Primary calli on CIM plate after 5 days of incubation at $32^{\circ} \mathrm{C}$ in continuous light. (B) Showing co-cultivated transformed calli after washing (Day 1) on selection medium. (C) Transformed calli after 14 days of incubation on selection Medium at $32^{\circ} \mathrm{C}$ in continuous light. (D) Showing transformed calli (day 1) on regeneration medium. (E) Showing emerging green plantlet after 45 days of incubation on regeneration medium at $28^{\circ} \mathrm{C}$ in 16 hours / 8 hours: light / dark condition respectively (Scale bar $\sim 1.0 \mathrm{~cm}$ ).
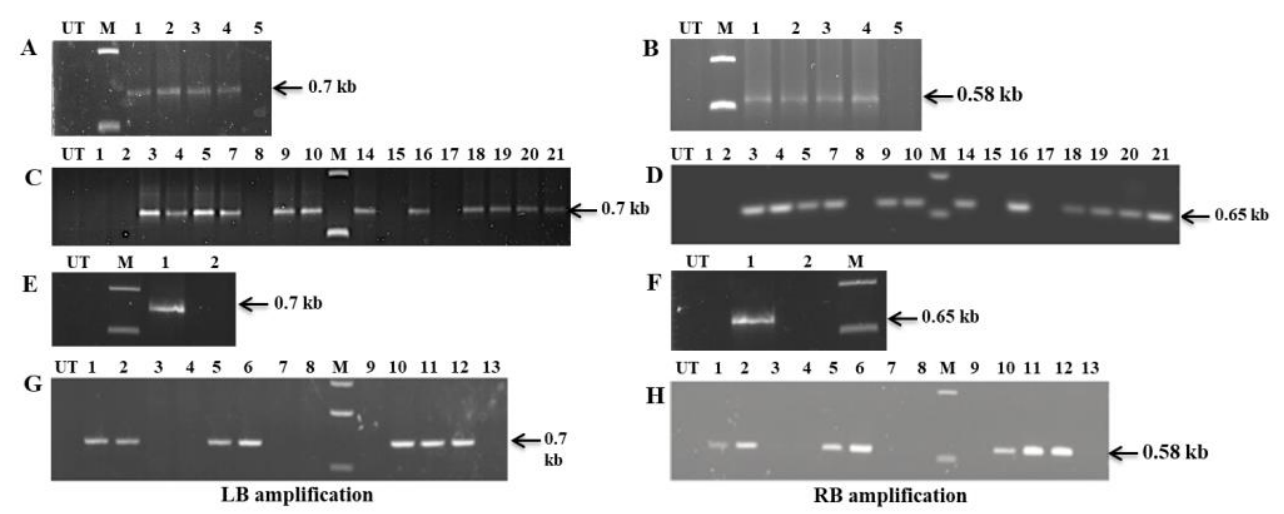

Fig 4. PCR analysis of the putative transgenic lines from all four different constructs. The T-DNA genomic fragment towards the left border (LB) in all the four constructs was amplified with primer 1 and 2. The T-DNA genomic fragments towards the right border (RB) was amplified with primer $(3,4)$ and $(4,5)$ in constructs $(\mathrm{I}, \mathrm{IV})$ and (II, III) respectively. (A-B) Putative transgenic plants from construct I showing PCR amplification in plant number 1, 2, 3, 4 and no amplification in plant number 5. (C-D) Putative transgenic plants from construct II showing PCR amplification in plant number 3, 4, 5, 7, 9, 10, 14, 16, 18, 19, 20, 21 and no amplification in plant number $1,2,8,15$ and 17 . (E-F) Putative transgenic plants from construct III showing PCR amplification in plant number 1 and no amplification in plant number 2. (G-H) Putative transgenic plants from construct IV showing PCR amplification in plant number 1, 2, 5, 6, 10, 11, 12 and no amplification in plant number 3, 4, 7, 8, 9,13. [Lane M - DNA Marker, Lane UT - untransformed PB1 plant]. 


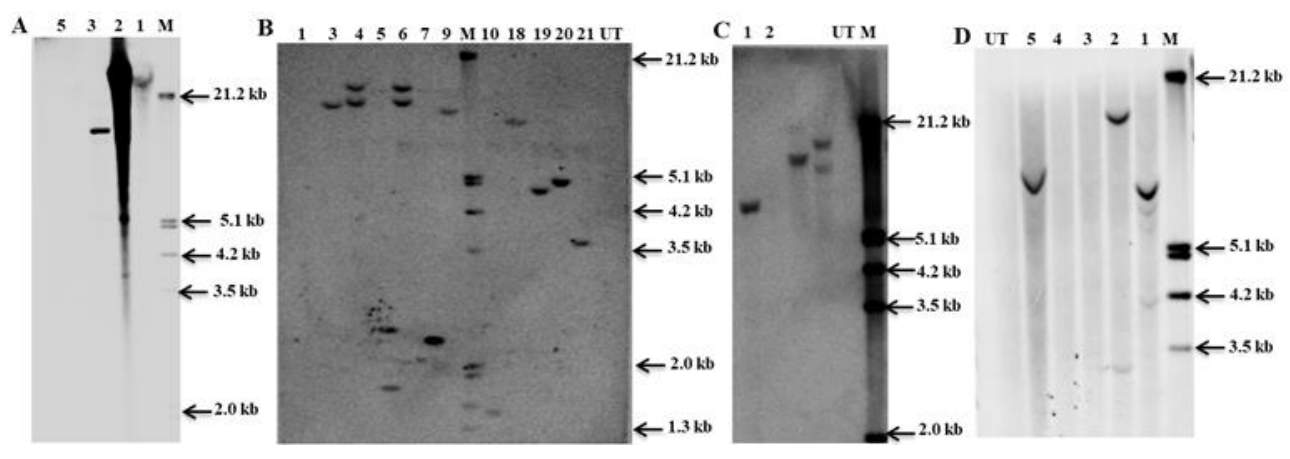

Fig 5. Southern blot analysis showing the presence or absence of transgenes in putative transgenic plants by using bar gene as probe. (A) Southern blot of Sbfl enzyme digested construct I putative transgenic plants (Plant number 1, 2 and 3 has transgene band whereas plant number 5 has no transgene band confirming transgenic escape). (B) Southern blot of Sbfl enzyme digested construct II putative transgenic plants (Plant number 1 has no transgene band confirming transgenic escape whereas plant numbers i.e. 3, 4, 5, 6, 7, 9, 10, 18, 19, 20 and 21 showing presence of transgene bands). (C) Southern blot of Sacl enzyme digested construct III putative transgenic plants (Plant number 1 has transgene band whereas plant number 2 has no transgene band confirming transgenic escape). (D) Southern blot of $\mathrm{Ncol}$ enzyme digested construct IV putative transgenic plants (Plant number 1 , 2 and 5 showing presence of transgene band whereas plant number 3 and 4 has no transgene band confirming transgenic escapes). [Lane M - DNA Marker, Lane UT - untransformed PB1 plant].

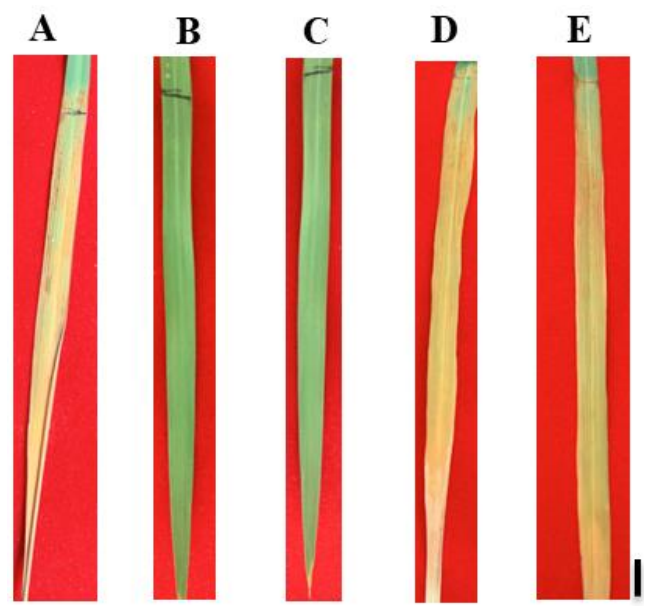

Fig 6. Leaves after Basta painting assay in putative transgenic lines for the final confirmation of transgenic escapes. (A) Control untransformed plant showing yellowing of leaf. (B-C) No yellowing in leaves in plant number 1 and 2 from construct IV. (D-E) Yellowing in leaves in plant number 3 and 4 from construct IV (Scale bar $\sim 1.0 \mathrm{~cm}$ ).

We also increased the incubation time in selection medium from 14 days (Toki et al., 2006) to 28 days for transformed calli, but again, there was no improvement. The PB1 transformation efficiency with PPT selection is significantly low in both methods of transformation as compared to hygromycin selection, where up to $46 \%$ transformation efficiency has been reported with 14 days old calli (Sahoo et al., 2011). The results suggested that bar gene is indeed poor selection system for PB1 cultivar.

Similar result had been also reported by Zhang et al. (2012) where the protocol using hptll gene as the selection marker in japonica rice cultivar, Kitaake saved 10 days, had double transformation efficiency and lower transgene copy number in comparison to the protocol using bar gene as the selection marker. Sohn et al. (2006) obtained 100\% cotransformed transgenic plants by using first, callus proliferation stage on hygromycin B and shoot regeneration stage on PPT selection, compared to $12.8 \%$ in the reverse selection condition. Thus, callus proliferation stage on PPT selection led to many transgenic escapes as seen also in our results. The result of PPT selection seems to be just opposite to hygromycin selection in both methods of transformations. Our result with PPT selection on PB1 cultivar is just opposite to that of Mannimaran et al. (2013), where around sevenfold increase in transformation efficiency was reported with hygromycin selection, when primary calli was used instead of secondary calli in the indica variety BPT 5204. If we compare transgenic escapes between hygromycin and PPT selection based transformation, then our in-house results (data not shown) along with published report suggests that there is almost no report of transgenic escape with hygromycin (Cheng et al., 1998; Sohn et al., 2006) 
irrespective of PPT selection, where transgenic escape is common even in other plant's transformation (Zeng et al., 2004; Zang et al., 2009).

The primary calli derived from mature seed's scutellum is compact and hard compared to secondary calli that is friable due to vigorous growth. Compact and hard nature of primary calli can lead to clear distinction between primary calli derived antibiotic-resistant secondary calli with untransformed calli after co-cultivation with Agrobacterium and resistant calli propagate independently. But, in the case of secondary calli due to its vigorous growth, distinction between resistant-to-transform and untransformed calli after co-cultivated with Agrobacterium becomes difficult and both types of calli can grow and propagate simultaneously (Saika and Toki, 2010). Therefore, primarycalli based transformation has more transformation efficiency compared to secondary-calli based transformation with hygromycin selection. With hygromycin selection, regeneration starts before callus browning (necrosis) because of the short period of callus proliferation and callus selection in primary calli based transformation, but condition is quite different in PPT selection. PPT selection is less stringent. Thus, non-transformed cells also grow in selection medium in large proportion, leading to transgenic escapes and less transformation efficiency. The more browning of callus in PPT selection may be the cause of less transformation efficiency (Zhao et al., 2008).

Advancement in the Agrobacterium transformation protocol is a continuous process and highly efficient protocol for rice transformation is very much required. The present study suggests that secondary calli as explants under PPT selection perform better than primary calli in the Agrobacteriummediated transformation of the indica PB1 rice.

\section{Materials and methods}

\section{Plant material}

Oryza sativa L. subspecies indica variety Pusa Basmati (PB1) was used for all the genetic transformation experiments. The seeds were obtained from Indian Agricultural Research Institute, New Delhi, India.

\section{Transformation vectors}

We have constructed four different binary vector constructs by routine recombinant DNA techniques. All the constructs were made in PPZP200 binary vector. The TOPO vector (pENTR) cloned fragments of promoter OsRTS (1.521 kb) and OsAgo3 $(1.760 \mathrm{~kb})$ were kind gifts from Prof. Sanjay Kapoor, University of Delhi, India. The TOPO vector (pENTR) cloned fragment of promoter OsFbox (1.974 kb) were kind gift from Dr. Reema Khurana, University of Delhi, India. Promoter OsG6b $(1.721 \mathrm{~kb})$ was synthesized commercially by Biolink (USA). The ubiquitin promoter was sub-cloned from pB4NU plasmid (Raghuvanshi, 2001). The TA cloned fragments of bar-poly $A$ and barstar-poly $A$ were kind gifts from Prof. Pradeep Kumar Burma, University of Delhi, India.

For construct I [(Ubi:bar::OsRTS:bs::OsAgo3:bs)], all the three expression cassettes such as (Ubiquitin - bar - poly A), (OsRTS- barstar - polyA) and (OsAgo3 - barstar - poly A) were made separately in TA cloning vectors and assembled sequentially in empty pPZP200 empty by sub-cloning (Fig
1A). For construct IV [(Ubi:bar::OsG6b:bs::OsAgo3:bs)], OsRTS promoter was replaced with OsG6b promoter by subcloning (Fig 1D). The construct II (Fig 1B) and III (Fig 1C) were made by modifying the [Ubi:bar::OsRTS:bn] binary vector, which were kind gift from Dr. Kulwinder Kaur, University of Delhi, India (Kaur, 2014). The OsRTS promoter from [Ubi:bar::OsRTS:bn] binary vector was replaced with OsG6b and OsFbox promoter by sub-cloning for construct II [(Ubi:bar::OsG6b:bn)] and III [(Ubi:bar::OsFbox:bn)] respectively.

\section{Plant transformation using secondary calli explants}

Rice transformation using secondary calli as explant with construct I and II were carried out following the highly accepted Hiei et al. (1994) protocol with few modifications. The seeds were dehusked and surface sterilized with $70 \%$ ethanol for $1 \mathrm{~min}, 0.1 \% \mathrm{HgCl}_{2}$ for $15 \mathrm{~min}, 1-2$ drops of mild detergent Tween-20 along with antifungal Bavistin and rinsed thoroughly in between with autoclaved double distilled water. The sterilized seeds were soaked in water overnight, blot dried on a layer of sterile tissue paper and inoculated on callus induction medium (CIM) [MS medium (Murashige and Skoog, 1962) containing B5 vitamins (Gamborg et al., 1976), 30 $\mathrm{g} \mathrm{I}^{-1}$ sucrose, $2 \mathrm{mg} \mathrm{I}^{-1} 2$ 4-D, $0.5 \mathrm{~g} \mathrm{I}^{-1}$ proline, $3 \mathrm{~g} \mathrm{I}^{-1}$ gelrite, $\left.\left.\mathrm{pH} 5.8\right)\right]$ in $90 \mathrm{~mm}$ petriplates incubated in dark at $28^{\circ} \mathrm{C}$ (Fig $2 \mathrm{~A}$ ).

After about 21 days, the scutellum - derived calli (Fig 2B) were sub-cultured on callus maintenance medium (CMM) [CIM, $1 \mathrm{~g} \mathrm{I}^{-1}$ casamino acids] (Fig 2C). Callus maintained for 34 days on $\mathrm{CMM}$ were infected with 0.1 O.D. secondary culture obtained from 36 hour grown primary culture of Agrobacterium tumefaciens strain EHA105 (Hood et al., 1993) harboring the binary vector construct and were cocultivated for 48 hour in dark at $28^{\circ} \mathrm{C}$ on co-cultivation medium [CMM, $10 \mathrm{~g} \mathrm{I}^{-1}$ glucose, $100 \mu \mathrm{M}$ acetosyringone]. Calli were washed thoroughly with sterile water containing amoxicillin $\left(250 \mathrm{mg} \mathrm{I}^{-1}\right)$ antibiotics, transferred to selection medium (SM) [CMM, $250 \mathrm{mg} \mathrm{l}^{-1}$ amoxicillin along with $2 \mathrm{mg} \mathrm{l}^{-}$ ${ }^{1}$ PPT and incubated at $28^{\circ} \mathrm{C}$ in dark. Calli growing on selection medium were sub-cultured thrice after 15 days interval (Fig 2D). The healthy proliferating calli growing on selection medium were transferred to regeneration medium (REM) [MS medium and B5 vitamins, $15 \mathrm{gl}^{-1}$ sucrose, $15 \mathrm{gl}^{-1}$ sorbitol, $2 \mathrm{mgl}^{-1}$ BAP, $0.5 \mathrm{mg} \mathrm{l}^{-1} \mathrm{NAA}, 250 \mathrm{mg} \mathrm{l}^{-1}$ amoxicillin, 3 $\mathrm{gl}^{-1}$ gelrite, $\mathrm{pH} \mathrm{5.8]} \mathrm{at} 28^{\circ} \mathrm{C}$ in 16 hours light / 8 hours dark photoperiod. Calli growing on REM were continuously subcultured in 15 days interval till green shoots emerged (Fig $2 \mathrm{E}$ ). Green shoots with $3-4 \mathrm{~cm}$ length (Fig 2F) were transferred to the rooting medium (RM) $[1 / 2 \mathrm{MS}$ and $\mathrm{B} 5$ vitamins, $2 \mathrm{~g} \mathrm{l}^{-1}$ gelrite]. Upon vigorous rooting in RM, the transgenic plantlets were grown in rice growth medium (Yoshida et al., 1976) for 1 - 2 weeks. Obtained transgenic plants were transferred to green house for optimum growth. This protocol takes around 4 - 5 months for transferring plants from laboratory to green house.

\section{Plant transformation using primary calli explants}

Rice transformation using primary calli as explant with construct III and IV was carried out following Toki et al. (2006) protocol with few modifications. Plant material and seed surface sterilization protocol is same as discussed in 
earlier method. The scutellum derived from 5-day-old cultured seedlings grown on N6D medium under continuous light at $32^{\circ} \mathrm{C}$ (Fig $3 \mathrm{~A}$ ) and were used for transfection with 0.1 O.D. secondary culture obtained from 36 hour grown primary culture of $A$. tumefaciens strain EHA105 harboring the binary vector construct and were co-cultivated for 3 days in dark at $25^{\circ} \mathrm{C}$.

After 3 days of co-cultivation at $25^{\circ} \mathrm{C}$ in the dark, a stringent washing of transformed seedlings were done with sterile water containing amoxicillin antibiotics $\left(250 \mathrm{mg} \mathrm{l}^{-1}\right)$ to remove Agrobacterium. The seeds were blot dried on a sterilized filter paper and cultured on selection medium (Fig 3B) [N6D medium containing $250 \mathrm{mg} \mathrm{I}^{-1}$ amoxicillin along

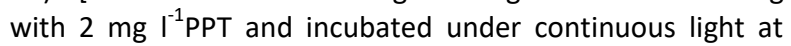
$32^{\circ} \mathrm{C}$ for 14 days (Fig $3 \mathrm{C}$ ). Selection medium survived, healthy growing transformed calli were transferred to REIII regeneration medium at $28^{\circ} \mathrm{C}$ in 16 hours light / 8 hours dark photoperiod (Fig 3D). Green shoots of $3-4 \mathrm{~cm}$ length were transferred to the HF rooting medium to induce roots (Fig $3 \mathrm{E})$. Obtained transgenic plants were transferred to green house for optimum growth. This transformation protocol took around 2-3 months with bar gene for transferring transgenic plants from laboratory to green house.

\section{Genomic DNA isolation and Southern blot analysis}

Genomic DNA was extracted from leaves of 1-2 month old transformed and untransformed control plants following the method of Dellaporta et al. (1983). Southern blotting analysis was performed according to standard protocols (Southern, 1975). $5 \mu \mathrm{g}$ genomic DNA samples were digested with restriction enzymes [construct I and II (Sbfl), construct III (SaCl) and construct IV (Ncol) (Fig1)] and resolved on $0.7 \%$ agarose gel overnight at 40 Volt. DNA from the treated gel was transferred overnight by capillary action to the nylon membrane, using 20X SSC buffer. The DNA was UV crosslinked to the membrane as per the manufacturer's instructions (HL-2000 HybriLinker Hybridization Oven, UVP). DNA probe (bar gene, Fig 1) was prepared using a PCR digoxigenin (DIG) probe synthesis Kit (Roche, Switzerland) according to the procedure recommended by the manufacturer. The hybridized DIG probes were immune detected with anti-DIG, Fab fragments conjugated to alkaline phosphatase and visualized with the chemiluminescence substrate CSPD by DIG Luminescent Detection Kit (Roche, Switzerland) with chemiluminescence visualization machine (Image Quant LAS 4000, GE healthcare Life science).

\section{PCR screening}

PCR was performed with 100 ng of template DNA with a thermal cycler (Eppendorf, Germany). The PCR reaction mixture $(20 \mu \mathrm{l})$ was prepared with genomic DNA, 1X Phusion buffer along with $0.5 \mu \mathrm{M}$ each of forward and reverse primers, $1.5 \mathrm{mM} \mathrm{MgCl}_{2}$, 3\% DMSO, $200 \mu \mathrm{M}$ dNTPs, and $1 \mathrm{U}$ of Phusion DNA polymerase (NEB). The PCR profile was set at $98^{\circ} \mathrm{C}$ for $30 \mathrm{~s}$ for initial denaturation, $35 \mathrm{cycles}$ of $\left(98^{\circ} \mathrm{C}\right.$ for $10 \mathrm{~s}, 62-68^{\circ} \mathrm{C}$ for $20 \mathrm{~s}, 72^{\circ} \mathrm{C}$ for $\left.30 \mathrm{~s}\right), 72^{\circ} \mathrm{C}$ for $10 \mathrm{~min}$. Primers used in these experiments are shown in Fig 1 and sequences are given in Supplementary Table 1.

\section{Expression analysis of bar gene in cassettes by Basta leaf} painting assay

The leaf of transgenic plant, including bar gene cassette in its genome, will grow normal (no yellowing of leaf) and untransformed control plant along with transgenic escapes will turn yellow after Basta leaf painting. The leaves (around $15-20 \mathrm{~cm}$ ) of the putative transgenic plants and untransformed control plants were painted with $0.05 \%$ Basta $\left(75 \mathrm{mgl}^{-1} \mathrm{PPT}\right)$. Leaf tissue response i.e. yellowing of leaves to PPT toxicity was scored after 1 week.

\section{Conclusion}

In conclusion, we found that secondary calli as explants perform better compared to primary calli under bar gene (PPT) selection during Agrobacterium-mediated indica rice transformation.

\section{Acknowledgements}

We thank Prof. Madan Mohan (University of Delhi South Campus) for providing lab support for our work. We thank Prof. Deepak Pental and Prof. Pradeep Kumar Burma (University of Delhi) for providing some constructs, which were modified in our work. We thank prof. Sanjay Kapoor (University of Delhi) for providing OsRTS promoter and OsAgo3 promoter. We also thank Dr. Kulwinder Kaur for providing [Ubi:bar::OsRTS:bn] construct and Dr. Reema Khurana (University of Delhi) for providing OsFbox promoter. PK would like to thank University Grant Commission, India for Dr. D.S. Kothari post-doctoral fellowship [File no. 13444/2011(BSR)] and Science and Engineering Research Board, Department of Science and Technology, India for Young Scientist Fast track grant (SB/YS/LS-104/2014). PK and RSP like to thank Guru Gobind Singh Indraprastha University, New Delhi, India and University of Delhi, India for support and guidance during project.

\section{Author contributions}

PK conceived and designed the experiments. PK and IC performed experiment. PK and RSP wrote the manuscript.

\section{References}

Altpeter F, Springer NM, Bartley LE, Blechl AE, Brutnell TP, Citovsky V, Conrad LJ, Gelvin SB, Jackson DP, Kausch AP, Lemaux PG, Medford JI, Orozco-Cardenas M, Tricoli DM, Eck JV, Voltas DF, Walbot V, Wang K, Zhang ZJ, Stewart CN (2016) Advancing crop transformation in the era of genome editing. Plant Cell. 28:1510-1520.

Burman N, Bhatnagar A, Khurana JP (2018) OsbZIP48, a HY5 transcription factor ortholog, exerts pleiotropic effects in light-regulated development. Plant Physiol. 176:1262-1285.

Chen YT, Fang QS, Chiang CH, Yeh SD, Wu HW Yu TA (2010) Transgenic Eustoma grandiflorum expressing the bar gene are resistant to the herbicide Basta. Plant Cell Tissue Organ Cult. 102(3):347-356.

Cheng X, Sardana R, Kaplan H, Altosaar I (1998) Agrobacteriumtransformed rice plants expressing synthetic $c r y \mid A(b)$ and $\operatorname{cry} I A(c)$ genes are highly toxic to striped stem borer and yellow stem borer. Proc Natl Acad Sci. 95:2767-2772. 
Dedicova B, Bermudez C, Prias M, Zuniga E, Brondani C (2014) High-throughput transformation pipeline for a Brazilian japonica rice with bar gene selection. Protoplasma. 252(4):1071-1083.

Dellaporta SL, Wood J, Hicks JB (1983) A plant DNA minipreparation: Version II. Plant Mol Biol Rep.1(4): 19-21.

Gamborg OL, Murashige T, Thorpe TA, Vasil IK (1976) Plant tissue culture media. In Vitro.12(7):473-478.

Hiei Y, Ishida Y, Komari T (2014) Progress of cereal transformation technology mediated by Agrobacterium tumefaciens. Front Plant Sci. 5:628.

Hiei Y, Komari T (2008) Agrobacterium-mediated transformation of rice using immature embryos or calli induced from mature seed. Nat Protoc. 3:824-834.

Hiei Y, Ohta S, Komari T, Kumashiro T (1994) Efficient transformation of rice (Oryza sativa L.) mediated by Agrobacterium and sequence analysis of the boundaries of the T-DNA. Plant J. 6(2):271-282.

Hood EE, Gelvin SB, Melchers LS, Hoekema A (1993) New Agrobacterium helper plasmids for gene transfer to plants. Transgenic Res. 2:208-218.

Kaur K (2014) Development of transgenic lines in rice for male sterility. Ph.D. Thesis, University of Delhi.

Khuong TTH, Crete P, Robaglia C, Caffarri S (2013) Optimisation of tomato microtom regeneration and selection on glufosinate/Basta and dependency of gene silencing on transgene copy number. Plant Cell Rep. 32:1441-145.

Lin YJ, Zhang Q (2004) Optimizing the tissue culture conditions for high efficiency transformation of indica rice. Plant Cell Rep. 23:540-547.

Lin D, Jiang Q, Ma X, Zheng K, Gong X, Teng S, Xu J, Dong Y (2018) Rice TSV3 encoding Obg-like GTPase protein is essential for chloroplast development during the early leaf stage under cold stress. Genes Genome Genetics. 8:253- 263.

Mahesh HB, Shirke MD, Singh S, Rajamani A, Hittalmani S, Wang G-L, Gowda M (2016) Indica rice genome assembly, annotation and mining of blast disease resistance genes. BMC Genomics. 17:242.

Mannimaran P, Ravi Kumar G, Raghurami Reddy M, Jain S, Bhaskar Rao T, Mangrauthia SK, Sundaram RM, Ravichandran S, Balachandran SM (2013) Infection of early and young tissues of indica rice BPT 5204 enhances regeneration and transformation efficiency. Rice Sci. 20(6):415-426.

Mehra P, Pandey BK, Giri J (2017) Improvement in phosphate acquisition and utilization by a secretory purple acid phosphatase (OsPAP21b) in rice. Plant Biotechnol J.15:10541067.

Murashige T, Skoog F (1962) A revised medium for rapid growth and bio-assays with tobacco tissue cultures. Physiol Plant. 15:473-497.

Nguyen VC, Nguyen VK, Singh CH, Devi GS, Reddy VS, Leelavathi $S$ (2017) Fast recovery of transgenic submergence tolerant rice cultivars of North - East India by early co-cultivation of Agrobacterium with pre-cultured callus. Physiol Mol Biol Plants. 23(1):115-123.

Raghuvanshi S (2001) Investigations on chloroplast transformation and characterization of constitively photomorphogenic1(COP1) gene in rice. Ph.D. Thesis, University of Delhi.

Ramesh S, Nagadhara D, Reddy VD, Rao KV (2004) Production of transgenic indica rice resistant to yellow stems borer and sapsucking insects, using super binary vectors of Agrobacterium tumefaciens. Plant Sci. 166:1077-1085.

Rao GS, Tyagi AK, Rao KV (2017) Development of an inducible male-sterility system in rice through pollen-specific expression of I-ornithinase (argE) gene of E.coli. Plant Sci. 256:139-147.

Reddy SSS, Singh B, Peter AJ, Rao TV (2016) Production of transgenic local rice cultivars (Oryza

sativa L.) for improved drought tolerance using Agrobacterium mediated transformation. Saudi J Biol Sci.:111.

Sah SK, Kaur A, Kaur G, Cheema GS (2014) Genetic transformation of rice: problems, progress and prospects. J Rice Res. 3:132.

Sahoo KK, Tripathi AK, Pareek A, Sopory SK, Singla-Pareek SL (2011) An improved protocol for efficient transformation and regeneration of diverse indica rice cultivars. Plant Methods. 7:49.

Singh RK, Prasad M (2016) Advances in Agrobacterium tumefaciens-mediated genetic transformation of graminaceous crops. Protoplasma. 253:691-707.

Saika H, Toki S (2010) Mature seed-derived callus of the model indica rice variety Kasalath is highly competent in Agrobacterium-mediated transformation. Plant Cell Rep. 29:1351-1364.

Sohn SI, Kim YH, Cho JH, Kim JK, Lee JY (2006) An efficient selection scheme for Agrobacterium-mediated cotransformation of rice using two selectable marker genes $h p t$ and bar. Korean J Breed. 38:173-179.

Southern EM (1975) Detection of specific sequences among DNA fragments separated by gel electrophoresis. J Mol Biol. 98:503-517.

Sun YW, Jiao GA, Liu ZP, Zhang X, Li JY, Guo XP, Du WM, Du JL, Francis F, Zhao YD, Xia LQ (2017) Generation of high-amylose rice through CRISPR/cas9-mediated targeted mutagenesis of starch branching enzymes. Front Plant Sci. 8:298.

Toki S, Hara N, Ono K, Onodera H, Tagiri A, Oka S, Tanaka H (2006) Early infection of scutellum tissue with Agrobacterium allows high-speed transformation of rice. Plant J. 47:969-976.

Toki S, Takamatsu S, Nojiri C, Ooba S, Anzai H, Iwata M, Christensen AH, Quail PH, Uchimiya H (1992) Expression of a maize ubiquitin gene promoter-bar chimeric gene in transgenic rice plants. Plant Physiol. 100:1503-1507.

Yoshida S, Forno DA, Cock JH, Gomez KA (1976) Routine procedures for growing rice plants in culture solution. In: Cock J H, Gomez K A. Laboratory Manual for Physiological Studies of Rice. Los Banos, Philippines: IRRI:61-66.

Zang N, Zhai H, Gao S, Chen W, He S, Liu Q (2009) Efficient production of transgenic plants using bar gene for herbicide resistance in sweet potato. Sci Horticult. 122:649-653.

Zeng P, Vadnais DA, Zhang Z, Polacco, JC (2004) Refined glufosinate selection in Agrobacterium mediated transformation of soybean [Glycine max (L.) Merrill]. Plant Cell Rep. 22:478-472.

Zhang CY, Li HY, Liu B (2012) A comparison study of hpt and bar as selection marker gene of transgenic rice. Hereditas (Beijing) 34(12):1599-1606.

Zhao LN, Zhou HJ, Lu LX, Liu L, Li XH, Lin YJ, Yu SB (2008). Identification of quantitative trait loci controlling rice mature seed culturability using chromosomal segment substitution lines. Plant Cell Rep. 28:247-256.

$\begin{array}{lcc}\text { ISAAA GM Approval } & \text { Database } \\ \text { http://www.isaaa.org/gmapprovaldatabase/ } & \end{array}$

